\title{
Insights into the Structure-Activity Relationship of Glycosides as Positive Allosteric Modulators Acting on P2X7 Receptors ${ }^{\text {s }}$
}

\author{
Waraporn Piyasirananda, Andrew Beekman, A. Ganesan, Stefan Bidula, \\ and (1) Leanne Stokes
}

School of Pharmacy, University of East Anglia, Norwich Research Park, Norwich, United Kingdom

Received July 21, 2020; accepted November 2, 2020

\begin{abstract}
$\mathrm{P} 2 \mathrm{X} 7$ is an important ligand-gated ion channel expressed in multiple immune cell populations. This study aimed to investigate the chemical requirements of triterpenoid glycosides within a new binding pocket to characterize the structure-activity relationship. A set of glycosides were screened for positive modulator activity at human $\mathrm{P} 2 \mathrm{X} 7$ using a YO-PRO-1 dye uptake assay in HEK-293 cells stably expressing the wild-type human P2X7 variant (HEK-hP2X7 cells). The highest positive modulator activity was with ginsenoside-compound $\mathrm{K}(\mathrm{CK})$, containing a monosaccharide (glucose) attached at carbon-20. Ginsenoside-20(S)-Rg3, containing a disaccharide group (glucose-glucose) at carbon-3, displayed positive modulator activity with a reduced $\mathrm{EC}_{50}$ for ATP and increased maximal response at human P2X7. The epimer 20(R)-Rg3 was inactive. A similar stereospecific pattern was observed for $20(S)-R h 2$. Ginsenoside-F1, highly similar to ginsenoside-CK but containing a single additional hydroxyl group, was also inactive at P2X7. Computational docking suggests hydrophobic residues in the pocket are involved in steric discrimination between triterpenoids, whereas the position and identity of the carbohydrate group are important for positive modulator activity at human P2X7. Ginsenosides containing
\end{abstract}

monosaccharide attachments perform better than di- or trisaccharide glycosides. Additional modifications to the triterpenoid scaffold at carbon- 6 are not tolerated. Gypenosides from plant sources other than Panax ginseng (gypenoside XVII, gypenoside XLIX, stevenleaf) can also act as positive allosteric modulators of P2X7. We also investigated the effect of positive allosteric modulators on endogenous P2X7 in THP-1 monocytes and confirmed our findings in a calcium response assay. A cell viability assay showed potentiation of ATP-induced cell death with ginsenoside-CK in THP-1 and HEK-hP2X7 cells.

\section{SIGNIFICANCE STATEMENT}

Ginsenosides are active as positive allosteric modulators at P2X7, and this study determines the chemical features important for mediating this effect. The position and identity of the sugar group is important for activity, as is the position of a number of hydroxyl groups on the triterpenoid scaffold. Diastereomers of ginsenoside-Rg3 and ginsenoside-Rh2 demonstrate the importance of the location of hydroxyl groups relative to the hydrophobic face of the predicted binding pocket.

\section{Introduction}

The ligand-gated ion channel P2X7 is important in regulating immune cell responses during infection and inflammation (Di Virgilio et al., 2017). In particular, P2X7 is a known physiologic regulator of the NLRP3-caspase 1 inflammasome complex and controls the secretion of proinflammatory cytokines such as interleukin-1 $\beta$ and interleukin-18 (Giuliani et al., 2017). Many studies have demonstrated that activation of P2X7 in infected macrophages in vitro can promote microbial killing. Intracellular bacteria and parasites such as Mycobacterium tuberculosis (Fairbairn et al., 2001, Placido et al., 2006), Toxoplasma gondii (Moreira-Souza et al., 2017), and Leishmania amazonensis (Chaves et al., 2014) are examples of pathogens in which P2X7 contributes to microbicidal

This work was supported by a Royal Thai government-funded $\mathrm{PhD}$ scholarship to W.P. and a BBSRC project grant to L.S. [BB/N018427/1].

https://doi.org/10.1124/molpharm.120.000129

S This article has supplemental material available at molpharm. aspetjournals.org. mechanisms. In vivo mouse models of infection suggest that global deficiency in P2X7 can affect pathogen burden and inflammation (Miller et al., 2015; Chaves et al., 2019). Furthermore, inheritance of loss-of-function variants of human P2X7 have been linked to susceptibility to infections and complications such as extrapulmonary tuberculosis (Fernando et al., 2007; Areeshi et al., 2015). Development of a positive allosteric modulator (PAM) of P2X7 may therefore be useful in treatment of such infections (Stokes et al., 2020). This type of therapy directed at enhancing host responses would reduce the need for antibiotics and be beneficial in avoiding development of antibiotic resistance.

Triterpenoid glycosides from Panax ginseng have PAM activity at human P2X7 with the in vivo metabolite ginsenoside-compound $\mathrm{K}$ (CK) demonstrating the highest activity (Helliwell et al., 2015). Using a combination of computational docking and mutagenesis, we previously characterized a novel binding pocket for ginsenosides within the central vestibule of human P2X7 (Bidula et al., 2019b). Predicted binding poses identified two modes of binding dependent on the carbohydrates attached

ABBREVIATIONS: AM, acetoxymetyl ester; CK, compound K; DMEM, Dulbecco's modified Eagle's medium; hP2X7, human P2X7; HEK-hP2X7 cell, HEK-293 cell stably expressing the wild-type hP2X7 variant; PAM, positive allosteric modulator; PPD, protopanaxadiol. 
to the ginsenoside in question. Ginsenoside-CK contains a single carbohydrate (glucose) moiety that makes multiple contacts with amino acids Asp318, Leu320 and Ser60. Conversely, ginsenoside-Rd contains both disaccharide (-glucose-glucose) and monosaccharide glucose moieties and is predicted to use the disaccharide moiety for binding to P2X7 (Bidula et al., $2019 \mathrm{~b})$. The presence of carbohydrate moieties is deemed essential for PAM activity since the aglycone protopanaxadiol has no activity at P2X7 (Helliwell et al., 2015).

In this study, we explored the chemical structural requirements of triterpenoid glycosides with the aim of building a structure-activity relationship for positive modulators at $\mathrm{P} 2 \mathrm{X} 7$. This revealed critical information about the tolerance of substitutions at carbon (C)-6 and the preference for glucose as the attached sugar moiety. This provides important knowledge for the future development of selective PAMs for P2X7.

\section{Materials and Methods}

Materials. Ginsenoside-CK (CAS 39262-14-1), ginsenoside-Rb1 (CAS 41753-43-9), ginsenoside-Rd (CAS 52705-93-8), 20(S)-ginsenoside-Rg3 (CAS 14197-60-5), 20(R)-ginsenoside-Rh2 (CAS 112246-158), protopanaxadiol (PPD) (CAS 30636-90-9), glycyrrhizic acid (CAS 1405-86-3), stevioside (CAS 57817-89-7), daucosterol (CAS 474-58-8), esculentoside A (CAS 65497-07-6), mogroside V (CAS 88901-36-4), saikosaponin A (CAS 20736-09-8), and stevenleaf (CAS 80321-63-7) were from Shanghai Richem International Ltd., China (supplier code CDCMANSETE). Ginsenoside-CK (CAS 39262-14-1), ginsenoside-F2 (CAS 62025-49-4), ginsenoside-F1 (CAS 53963-43-2), gypenoside XLIX (CAS 94987-08-3), gypenoside XVII (CAS 80321-69-3), 20(S)ginsenoside-Rg3 (CAS 14197-60-5), 20(R)-ginsenoside-Rg3 (CAS 38243-03-7), 20(S)-ginsenoside-Rh2 (CAS 78214-33-2), and 20(R)ginsenoside-Rh2 (CAS 112246-15-8) were from ChemFaces (Wuhan Chemfaces). Scilliroside (CAS 507-60-8, NSC7523), ouabain (CAS 63060-4, NSC25485), solanine hydrochloride (CAS 20562-02-1, NSC35611), and solasonine (CAS 19121-58-5, NSC82149) were obtained from the National Cancer Institute Developmental Therapeutics Program chemical repository. ATP (A7699; Sigma-Aldrich) was dissolved in distilled water to $100 \mathrm{mM}$ and adjusted to $\mathrm{pH} 7.4$ with $5 \mathrm{M} \mathrm{NaOH}$. Aliquots of ATP were kept frozen at $-20^{\circ} \mathrm{C}$ and used once in experiments. AZ10606120 (CAS 607378-18-7; Tocris Biosciences) and AZ11645373 (CAS 227088-94-0; Sigma-Aldrich) were dissolved in DMSO to $10 \mathrm{mM}$ and stored at $-20^{\circ} \mathrm{C}$. Fura- 2 acetoxymethyl ester (AM) (CAS 108964-32-5; HelloBio) was prepared in DMSO to a concentration of $1 \mathrm{mM}$, aliquoted, and stored at $-20^{\circ} \mathrm{C}$ in amber-colored Eppendorf vials. Sulfinpyrazone (CAS 57-96-5; Sigma Aldrich) was dissolved in methanol at a stock concentration of $25 \mathrm{mM}$ and stored at $+4^{\circ} \mathrm{C}$.

Cell Culture. HEK-293 cells stably expressing the wild-type human P2X7 (hP2X7) variant (HEK-hP2X7) were maintained in Dulbecco's modified Eagle's medium (DMEM):F12 (catalog number 11320-074; ThermoFisher Scientific, Life Technologies) containing $10 \%$ fetal bovine serum (catalog number 10500-064; Gibco) and penicillin/streptomycin (ThermoFisher Scientific) as described previously (Helliwell et al., 2015). HEK-293 cells were passaged twice weekly using $0.25 \%$ trypsin-EDTA (ThermoFisher Scientific). THP-1 human monocytic cells (a kind gift from Professor Maria O'Connell, University of East Anglia) were maintained in RPMI 1640 medium (catalog number 21875-034, Fisher Scientific, Life Technologies) containing $10 \%$ fetal bovine serum (Gibco, as before) and penicillin/streptomycin (ThermoFisher Scientific). Cells were kept in a humidified incubator at $37^{\circ} \mathrm{C}$ with a constant supply of $5 \% \mathrm{CO}_{2}$.

YO-PRO-1 Dye Uptake Screening Assay. ATP-induced dye uptake experiments were performed as described previously (Bidula et al., 2019b). Briefly, HEK-hP2X7 cells were plated at $2 \times 10^{4}$ cells per well $(100 \mu \mathrm{l}$ per well) in complete medium and left overnight to attach to poly-D-lysine-coated 96 -well plates. YO-PRO-1 iodide was prepared in a low divalent buffered solution $(145 \mathrm{mM}, 2 \mathrm{mM} \mathrm{KCl}, 13 \mathrm{mM}$ D-glucose, $10 \mathrm{mM}$ HEPES, $0.2 \mathrm{mM} \mathrm{CaCl}_{2} \mathrm{pH}$ 7.3) to a final concentration of $2 \mu \mathrm{M}$. Medium was removed from the plate using a manual multichannel pipette and YO-PRO-1 containing buffer applied to wells (180 $\mu \mathrm{l}$ per well). AZ10606120 $(10 \mu \mathrm{M})$ was prepared in YO-PRO-1 low divalent buffer and added directly to the cells to block P2X7 by pretreatment. A Flexstation 3 plate reader (Molecular Devices, Sunnyvale, CA) was used to record YO-PRO-1 fluorescence in HEKhP2X7 for 300 seconds after the addition of ATP (at $10 \times$ final concentration) with/without ginsenoside compounds or vehicle (DMSO). Plates were allowed to warm up to $37^{\circ} \mathrm{C}$ for 10 minutes before initiating the recording. Baseline recordings were made for 40 seconds before compound addition using the Flexstation 3 fluidics system. YO-PRO-1 fluorescence was measured at $520 \mathrm{~nm}$ after excitation at $490 \mathrm{~nm}$ (auto cutoff at $495 \mathrm{~nm}$ ), and the sample interval was 3.5 seconds. Flexstation settings were photomultiplier tube (PMT) medium, six reads per well, pipette height 170, and a rate of injection of 3. Data were acquired using Softmax Pro version 5.4 (Molecular Devices). To analyze the data, area under the dye uptake curve was calculated in Softmax Pro version 5 using zero baseline data with a lag time of 50 seconds (50-300 seconds).

Fura-2 Calcium Measurements. THP-1 cells were pelleted by centrifugation ( $300 \mathrm{~g}, 5$ minute), washed, and resuspended in Hanks' balanced salt solution buffer containing $2 \mu \mathrm{M}$ fura-2 AM (HelloBio) and $250 \mu \mathrm{M}( \pm)$-sulfinpyrazone. Cells were loaded for 1 hour in a waterbath at $37^{\circ} \mathrm{C}$ while shielded from light with foil. Loaded cells were washed once with $5 \mathrm{ml}$ Hanks' balanced salt solution to remove excess fura-2 AM dye. THP-1 cells were then resuspended, counted, and plated at $2 \times 10^{5}$ cells per well in a standard clear 96 -well plate (NUNC 167008; Thermo Scientific) in low divalent assay buffer without $\mathrm{Mg}^{2+}\left(145 \mathrm{mM} \mathrm{NaCl}, 2 \mathrm{mM} \mathrm{KCl}\right.$ and $2 \mathrm{mM} \mathrm{CaCl}_{2}, 13 \mathrm{mM}$ glucose, $10 \mathrm{mM}$ HEPES, $\mathrm{pH}$ 7.3) containing $250 \mu \mathrm{M}$ (土)-sulfinpyrazone. The $10 \times$ concentration of agonist (ATP) was prepared in the same assay buffer. ATP was injected automatically into wells at 30 seconds in a Flexstation 3 microplate reader. Ratiometric data were acquired using 340 and $380 \mathrm{~nm}$ for excitation wavelengths, $520 \mathrm{~nm}$ as the emission wavelength, and six reads per well (PMT medium). Area under the curve was calculated using standard zero baseline normalization with a lag time 0-300 seconds using SoftMax Pro version 5.4 software.

Cell Viability Assay. HEK-hP2X7 or THP-1 cells were seeded at $5 \times 10^{3}$ cells per well in a volume of $100 \mu \mathrm{l}$ for 24 hours in 96 -well plates (Nunclon Edge plates, catalog number 167425; Thermo Scientific). Cells were plated in DMEM:F12 medium or RPMI 1640 medium containing 1\% FBS and penicillin/streptomycin for 24 hours in a humidified incubator at $37^{\circ} \mathrm{C}$ with a constant supply of $5 \% \mathrm{CO}_{2}$. Edge wells of the plates were filled with autoclaved distilled water to prevent evaporation of media from wells containing cells. The compounds and ATP were made at $2 \times$ final concentration in $1 \%$ FBS DMEM:F12 and $100 \mu \mathrm{l}$ added to the cells for 24 hours. After incubation, resazurin sodium salt (CAS 62758-13-8; Sigma Aldrich) at $0.1 \mathrm{mg} / \mathrm{ml}$ in sterile PBS was added to the plate $(20$ into $200 \mu \mathrm{l}$ culture medium per well). Cells were incubated for a further 2 hours in a humidified incubator at $37^{\circ} \mathrm{C}$ with a supply of $5 \% \mathrm{CO}_{2}$. A Flexstation 3 microplate reader was used to acquire data. Endpoint fluorescence data were measured using $570 \mathrm{~nm}$ excitation wavelength, $600 \mathrm{~nm}$ emission wavelength (cutoff at $590 \mathrm{~nm}$ ), and three reads per well (PMT low). Data were analyzed by performing background correction through subtracting blank medium readings (without cells) from all samples and then normalizing each sample to medium treated cells (control treatment, 100\%) as percentage of control.

Computational Docking. The homology model of human P2X7 generated previously in (Bidula et al., 2019b) was used for docking runs using the Schrödinger Maestro suite. Three-dimensional models of ginsenoside-F1 and ginsenoside-20(S)-Rg3 were generated using LigPrep software, and the OPLS3 force field was used to generate up to 32 low-energy conformers for each ligand. Induced-fit docking was 
performed using the automated extended sampling protocol, first performing several initial docking runs in which either side chains were trimmed or van der Waals potentials were softened according to their flexibility; then side chains were rebuilt, and those within $5 \AA$ of the ligand were optimized using Prime software (Jacobson et al., 2004). Structures within $30 \mathrm{kcal} \mathrm{mol}^{-1}$ of the lowest energy structure were retained. Ligands were then redocked to the new receptor structure using the Glide SP algorithm (Friesner et al., 2004) and standard potentials. The receptor grid was centered on the highest scoring potential binding site using SiteMap (Halgren, 2007), and this had a cubic box with dimensions of $30 \AA$. For each ginsenoside the resulting poses were clustered by heavy atom root-mean-square deviation using the average-linkage method, and a representative structure was chosen from the model closest to the centroid of the most populated cluster. For ginsenoside-F1 and 20(S)-Rg3 the most populated cluster made up $31 \%$ and $62 \%$ of all solutions, respectively.

Data and Statistical Analysis. Results are expressed as means \pm S.D. from the indicated number of experiments. For YO-PRO-1 dye uptake and intracellular calcium experiments, each independent experiment used triplicate wells, and the means of the replicates were collated and plotted. Technical replicates were used to ensure reliability of fluorescence values. Cell viability data were normalized to the vehicle control for each experiment after background correction had been performed. Dose-response curves were plotted by a nonlinear regression fit with variable slope using GraphPad Prism software version 7. Half-maximal responses are expressed as $\mathrm{EC}_{50}$ values with
95\% confidence intervals. These values were calculated from the collated data for each compound from three independent experiments. Statistical differences were determined by analysis of the data by oneway ANOVA followed by Dunnett's multiple comparison test or Sidak's multiple comparison test using GraphPad Prism version 7. $P<0.05$ was the accepted minimum level of significance.

\section{Results}

To measure positive modulator activity at human P2X7 receptors, we used a well characterized HEK-hP2X7 stable cell line (Helliwell et al., 2015) and a YO-PRO-1 dye uptake assay performed on a Flexstation 3 plate reader. Figure 1A shows a typical dye uptake response to an approximate $\mathrm{EC}_{50}$ concentration of agonist (200 $\mu \mathrm{M}$ ATP) and the effect of coadministration of ginsenoside-CK or ginsenoside-Rd at a final concentration of $10 \mu \mathrm{M}$. We previously demonstrated that pretreatment with a selective P2X7 antagonist AZ10606120 abolished the response, confirming that the effect of the ginsenosides was dependent on P2X7 activation (Helliwell et al., 2015). Dose-response experiments demonstrated that ginsenoside-CK and ginsenoside-Rd have two effects on ATP-induced responses at hP2X7 (Fig. 1B): an increase in the maximum response (a type I PAM effect) and
A

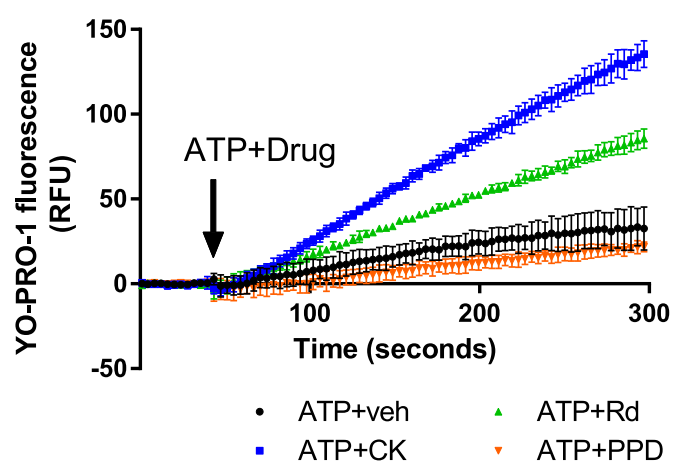

B

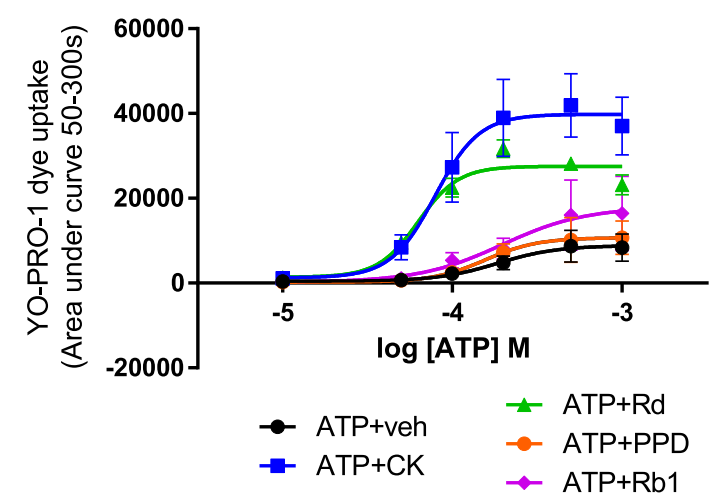

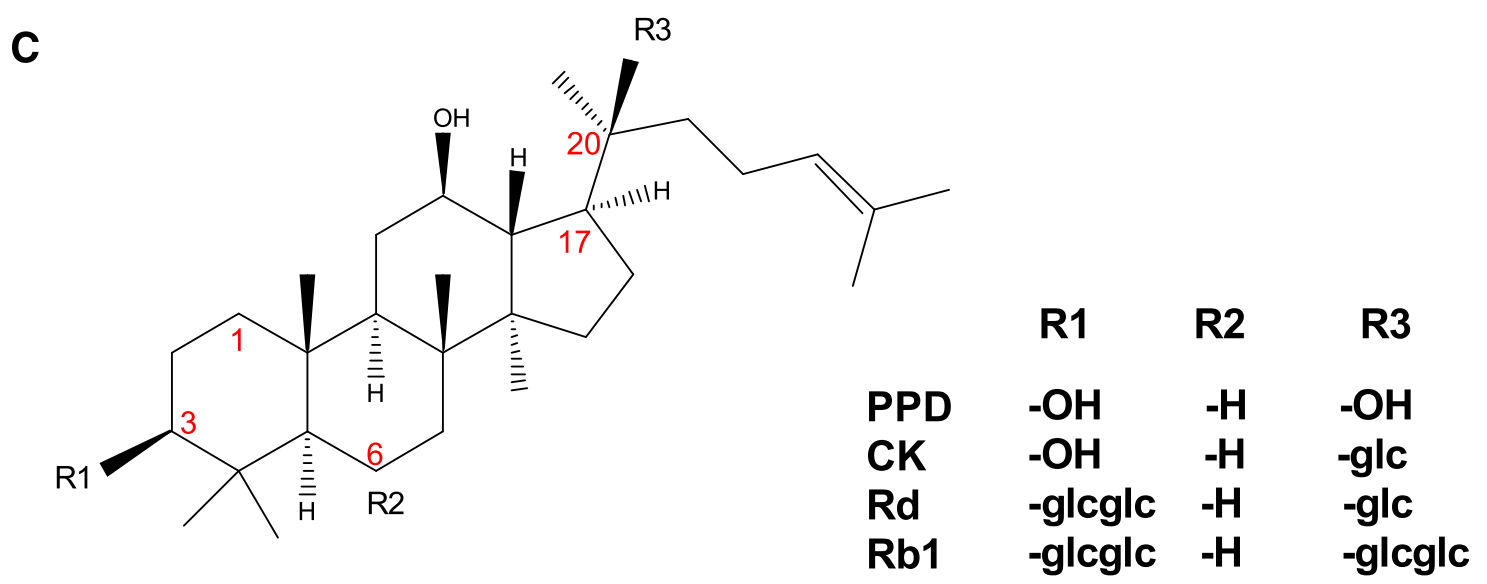

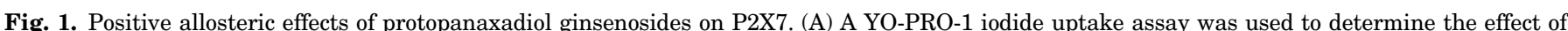

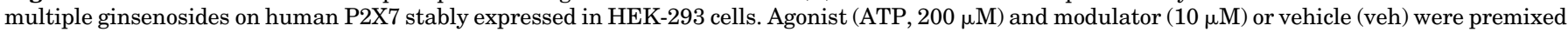

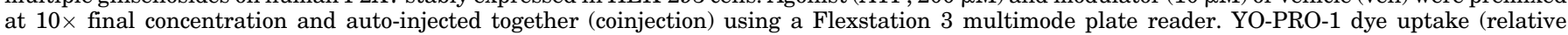

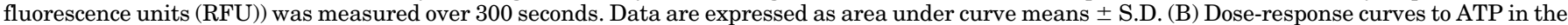

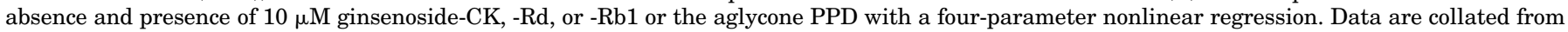

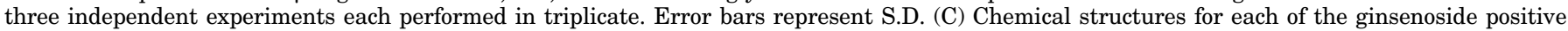
modulators and the inactive aglycone PPD are shown. 
a shift in the dose-response curve to the left (a type II PAM effect), thereby enhancing the maximum effect of the agonist and reducing the $\mathrm{EC}_{50}$ value respectively. Classification of type I, type II and type I/II mixed PAM effects have been previously used for $N$-methyl-D-aspartate (NMDA) receptors (Hackos and Hanson, 2017), and the same naming convention for P2X receptors is discussed in a recent review (Stokes et al., 2020). Ginsenoside-CK reduced the $\mathrm{EC}_{50}$ for ATP to $61.6 \mu \mathrm{M}$ compared with an average $\mathrm{EC}_{50}$ for ATP + vehicle (DMSO) of
$219 \mu \mathrm{M}$ ( $n=5$ experiments) and increased the maximum response by 2.4-4.4-fold (Table 1 ). Both ginsenoside-CK and ginsenoside-Rd have mixed type I/II effects. Ginsenoside-Rb1 increased the maximum response by 1.9 -fold but had little effect on the $\mathrm{EC}_{50}$ value $(175.9 \mu \mathrm{M}$; Fig. 1B) and therefore has only type I PAM activity. The aglycone ginsenoside metabolite PPD had no effect on the ATP dose-response curve (Fig. 1B).

Investigating Glycosylation Patterns. We assumed that the carbohydrate groups play a key role in mediating
A

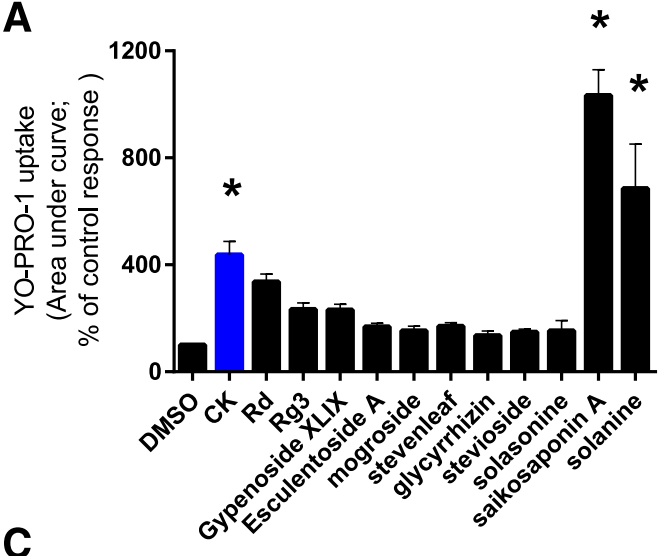

B

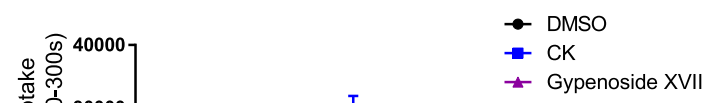

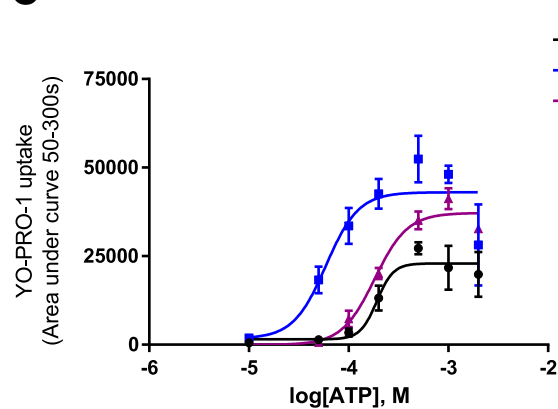

E

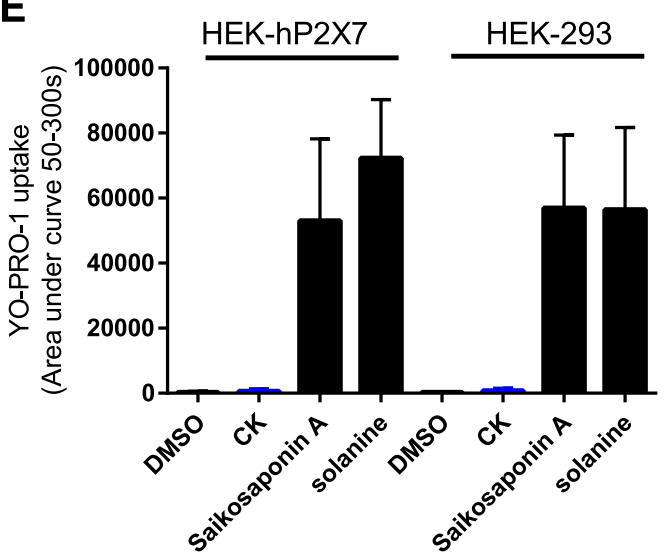

$$
\rightarrow \text { DMSO }
$$$$
\rightarrow \mathrm{CK}
$$$$
\text { - Gypenoside XLIX }
$$

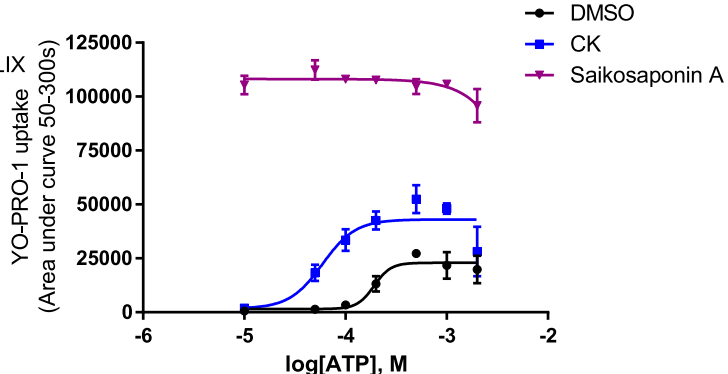

$\mathbf{F}$

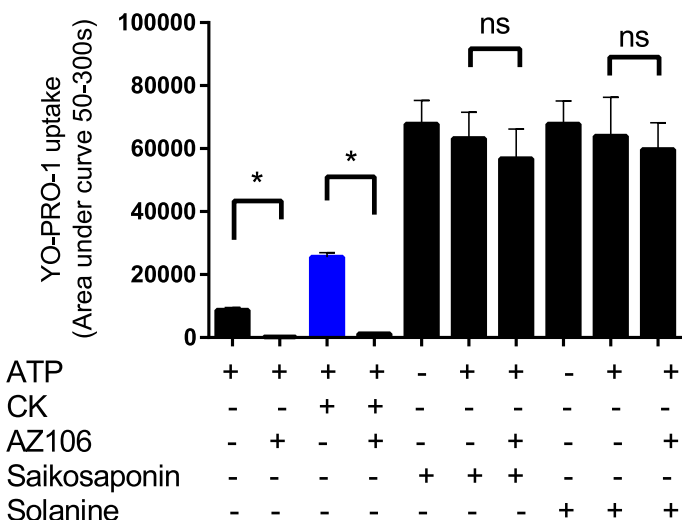

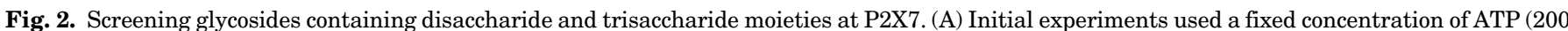

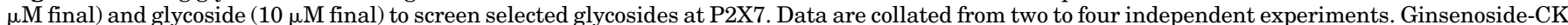

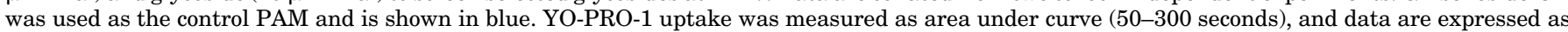

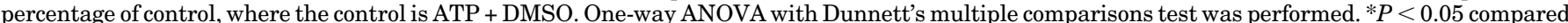

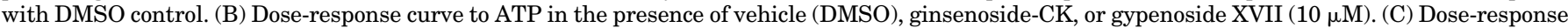

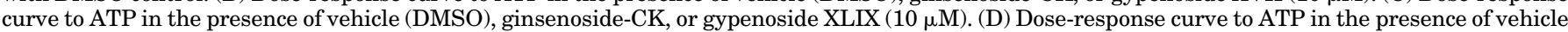

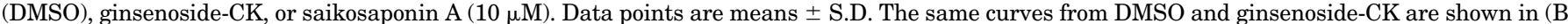

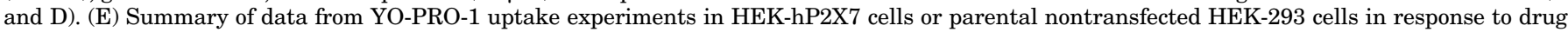

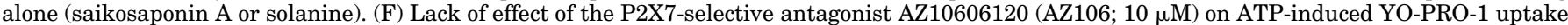

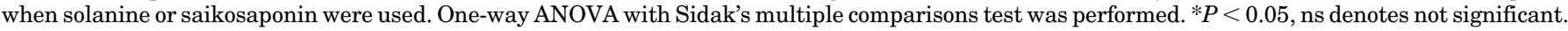




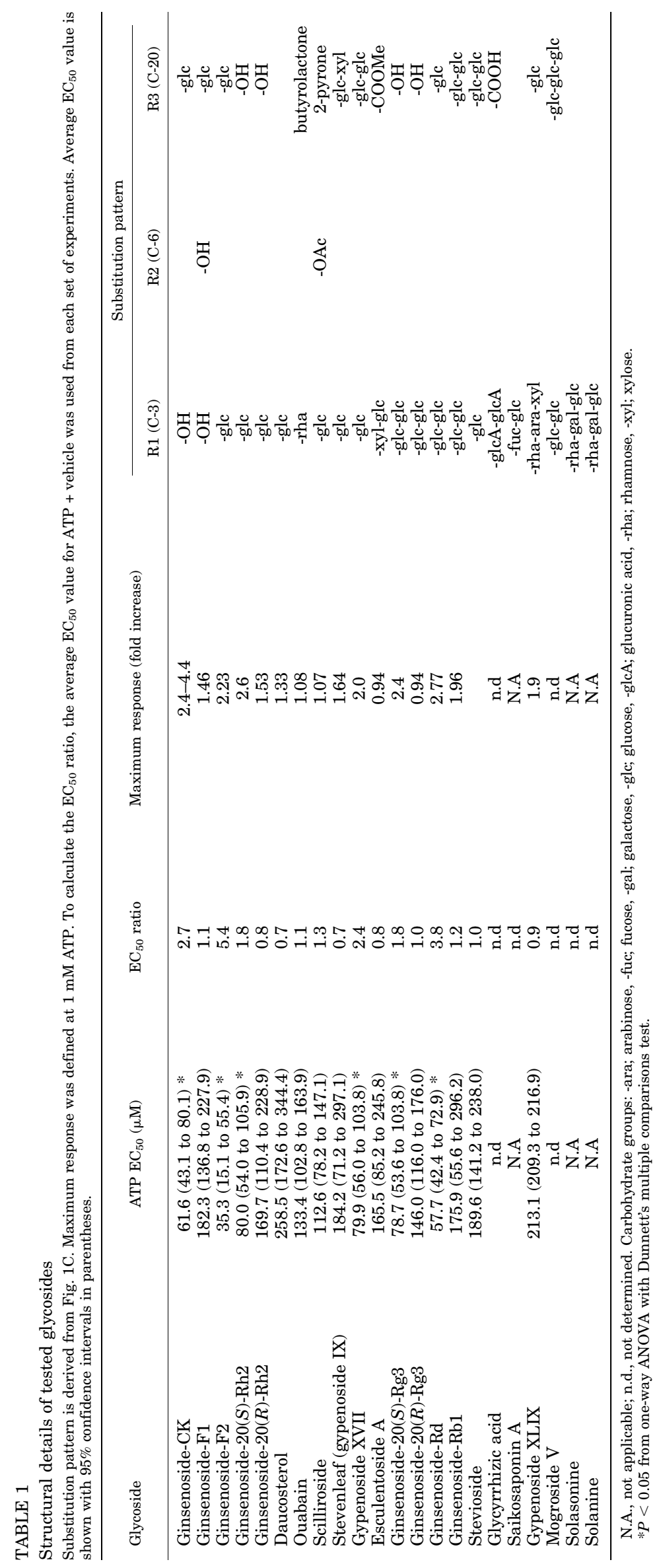


this response at P2X7 since the aglycone PPD had no effect (Fig. 1B). Our previous study identified a binding site within the human P2X7 trimeric structure based on computational docking to a homology model. The single glucose moiety on ginsenoside-CK makes predicted interactions with P2X7 $\beta$ strands lining the lateral portals which connect the agonistbinding site to the ion channel transmembrane domains (Bidula et al., 2019b). We investigated the effect of varying the number of sugar moieties attached to the steroid-like scaffold by searching for chemicals similar to ginsenosides that were commercially available as purified chemicals. We excluded ginsenosides from the protopanaxatriol series with sugars attached on C- 6 as our previous work has demonstrated these compounds to have no activity at P2X7 (Helliwell et al., 2015). We identified a number of candidate glycosides to test (Table 1) and screened these compounds at a final concentration of $10 \mu \mathrm{M}$ on $\mathrm{hP} 2 \mathrm{X} 7$ responses using a fixed concentration of ATP $(200 \mu \mathrm{M})$ in the YO-PRO-1 dye uptake assay. Focusing on glycosides containing a disaccharide moiety first (Fig. 2) we found that ginsenoside-Rg3 (mixed isomers) and gypenoside XLIX showed a small increase in the ATP-induced YO-PRO-1 response, which was not statistically significant (Fig. 2A), whereas saikosaponin A and solanine both showed a large increase in the ATP-induced YO-PRO-1 dye uptake response (Fig. 2A). Most other glycosides in this category showed no modulation of P2X7 (esculentoside A, mogroside V, glycyrrhizic acid, and stevioside). Stevenleaf showed a minor modulation increasing the maximum response only (1.64-fold; Supplemental Fig. 1). Dose-response experiments demonstrated that gypenoside XVII has a type II PAM activity at P2X7 (Fig. 2B), reducing the $\mathrm{EC}_{50}$ value for ATP (Table 1) and increasing the maximum response by 2fold. In contrast, gypenoside XLIX did not reduce the $\mathrm{EC}_{50}$ value for ATP (Table 1) but did increase the maximum response by 1.9 -fold (Fig. 2C). Further investigations into saikosaponin A (Fig. 2D) revealed that this glycoside
A

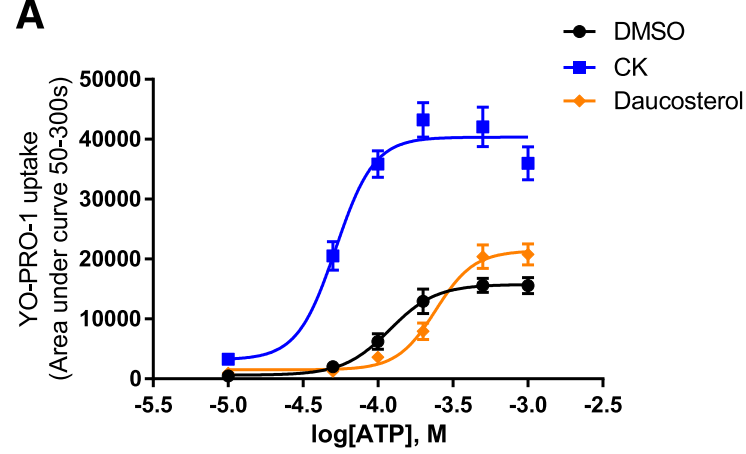

C

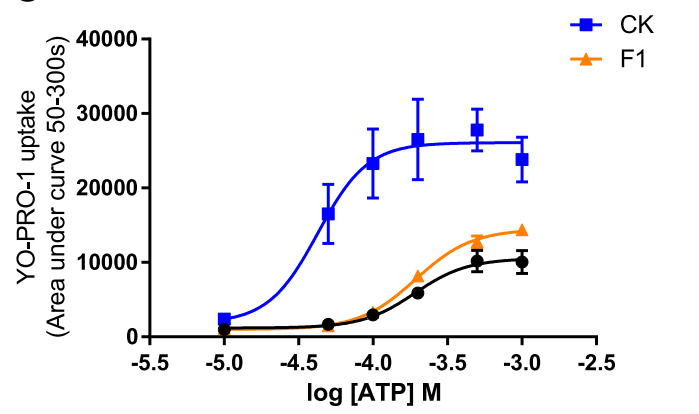

E

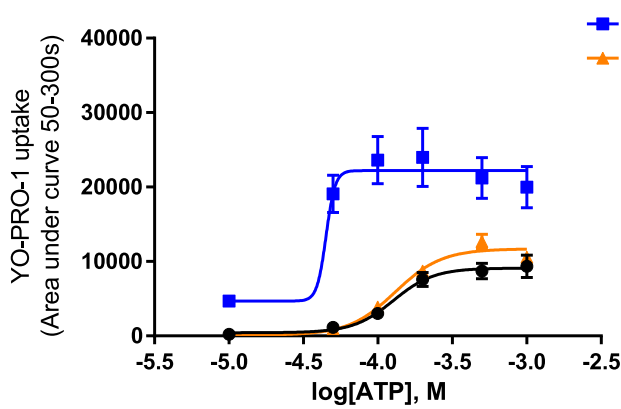

B

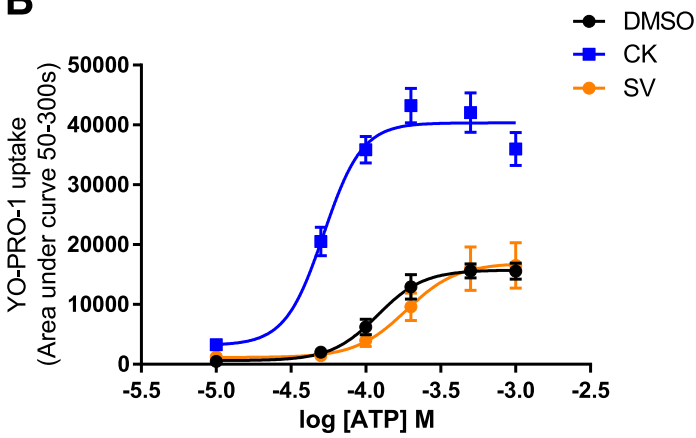

D

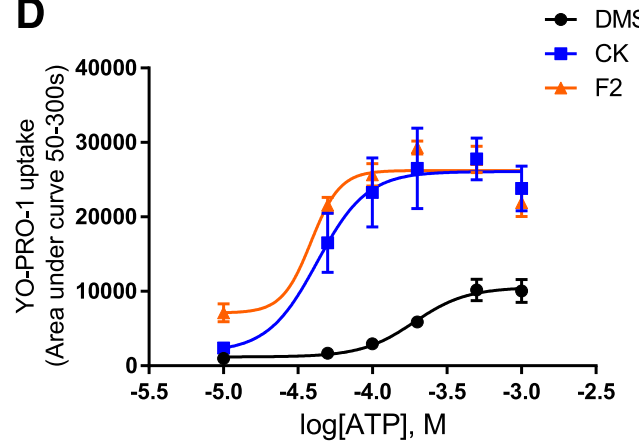

$\mathbf{F}$

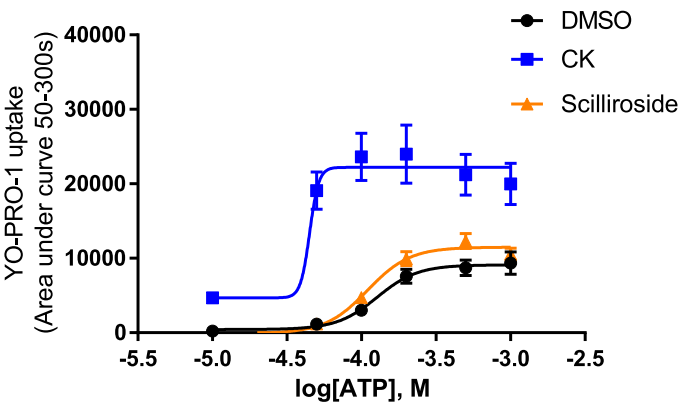

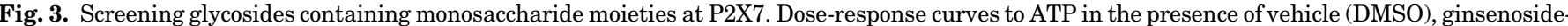

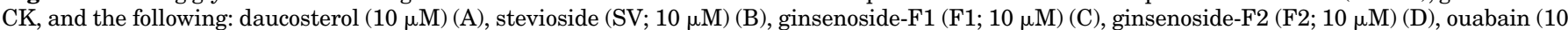

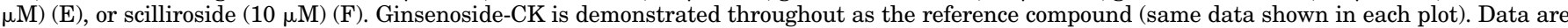
collated from three independent experiments each performed in triplicate. Error bars represent S.D. 
increased YO-PRO-1 uptake at all concentrations of ATP tested and could induce YO-PRO-1 uptake in nontransfected HEK-293 cells (which have no expression of P2X7) and in HEK-hP2X7 cells in the absence of ATP (Fig. 2E), suggesting that this effect was not P2X7-dependent. We confirmed this by pretreating HEK-hP2X7 cells with a P2X7-selective antagonist, AZ10606120. ATP-induced responses were abolished in cells treated with $10 \mu \mathrm{M}$ AZ10606120, as were responses induced by ATP + ginsenoside-CK (Fig. 2F). However, responses to ATP + saikosaponin A or ATP + solanine were unaffected by AZ10606120 pretreatment, suggesting that P2X7 was not involved (Fig. 2F).

We then investigated glycosides with monosaccharide attachments including daucosterol, ginsenoside-F2, ginsenoside-F1, ouabain, and scilliroside (Table 1). Dose-response experiments demonstrated that ginsenoside-F2 could both increase the maximum response and reduce the $\mathrm{EC}_{50}$ value for ATP (Table 1) and displayed a similar mixed type I/II effect as ginsenoside-CK (Fig. 3D). Ouabain, daucosterol, and scilliroside had no effect on the ATP dose-response curves (Fig. 3). Interestingly, ginsenoside-F1, which has an almost identical chemical structure to ginsenoside-CK, did not potentiate P2X7 responses (Fig. 3C). The presence of one additional hydroxyl group on C-6 is the only difference between ginsenoside-CK and ginsenoside-F1, and this could potentially interfere with correct positioning into the $\mathrm{P} 2 \mathrm{X} 7$ binding pocket.

Computational Docking. To investigate the predicted theoretical docking differences between ginsenoside-CK and ginsenoside-F1, we used computational docking to a homology model of human P2X7 (Bidula et al., 2019b). Replacing ginsenoside-CK (Fig. 4A) in the open ATP-bound model of $\mathrm{hP} 2 \mathrm{X} 7$ with ginsenoside-F1 resulted in an analogous predicted pose (Fig. 4B) and this would orient the additional hydroxyl group on C- 6 to be facing the hydrophobic side of the pocket close to L320 and F322. Since ginsenoside-F1 has no activity at P2X7, this purely theoretical pose suggests that steric hindrance and a repulsive effect would prevent ginsenoside-F1 from interacting with the PAM site at P2X7. Furthermore, there would likely be an energy penalty due to poor solvation of the additional $\mathrm{OH}$ group within the hydrophobic pocket.

Concurrently we investigated chemicals thought to bind in an inverted mode such as ginsenoside-Rd (Bidula et al., 2019b). Ginsenosides with sugars attached to C-3 rather than C-20 are predicted to insert sugars deep into the pocket and would have the C-20 side chain solvent-exposed. We have previously demonstrated that the monosaccharide ginsenoside$\mathrm{Rh} 2$ is able to potentiate $\mathrm{hP} 2 \mathrm{X} 7$ responses (Helliwell et al., 2015) and have proposed a predicted binding model (Bidula et al., 2019b). Ginsenoside-Rh2 and ginsenoside-Rg3 are also predicted to bind in this inverted mode, and both of these ginsenosides can exist as two diasteromers with regard to the C-20 side chain. The natural product form in $P$. ginseng is believed to be the 20(S)-diastereomer (Qi et al., 2011; Yang et al., 2014), and this is thought to have higher bioactivity than the 20(R)-diastereomer (Wei et al., 2012). We investigated the individual pure diastereomers to determine if this had any bearing on PAM activity at P2X7 and found that only the 20(S)-diastereomers of Rg3 and Rh2 are active as PAMs at hP2X7 (Fig. 5). This suggests that the stereospecific positioning of the $-\mathrm{OH}$ group on $\mathrm{C}-20$ may be critical for mediating the potentiating effect. To investigate which part of P2X7 would be closest to C-20, we used computational induced-fit docking using Glide as previously reported (Bidula et al., 2019b). The most populated pose for 20(S)Rg3 is presented in Fig. 6, and this places the C-20 hydroxyl group pointing away from the hydrophobic side of the pocket. In an analogous theoretical pose, $20(R)$-Rg3 would have this C-20 hydroxyl group in close proximity to the hydrophobic face, increasing steric hindrance and repulsive effects plus the desolvation penalty as mentioned above, preventing 20(R)-Rg3 and 20(R)-Rh2 from interacting with the binding pocket on P2X7.

Modulation of Endogenous Human P2X7 in Immune Cells. Finally, we verified our findings on the identified active versus inactive glycosides by using a human THP-1 monocytic cell line known to endogenously express P2X7 (Fig. 7). Using fura-2 AM loaded cells, we measured ATPinduced calcium responses (Fig. 7A) and found that there was a rapid peak increase in calcium followed by a sustained elevation of calcium over 300 seconds of recording. Pretreatment of THP-1 cells with commercially available P2X7selective antagonists such as AZ11645373 or AZ10606120 did not dramatically affect the response to $500 \mu \mathrm{M}$ ATP (Fig. 7), but coapplication of ginsenoside-CK with $500 \mu \mathrm{M}$ ATP increased the peak response and the sustained elevation in $\left[\mathrm{Ca}^{2+}\right]_{\mathrm{i}}$ (Fig. 7). This increased response could be completely

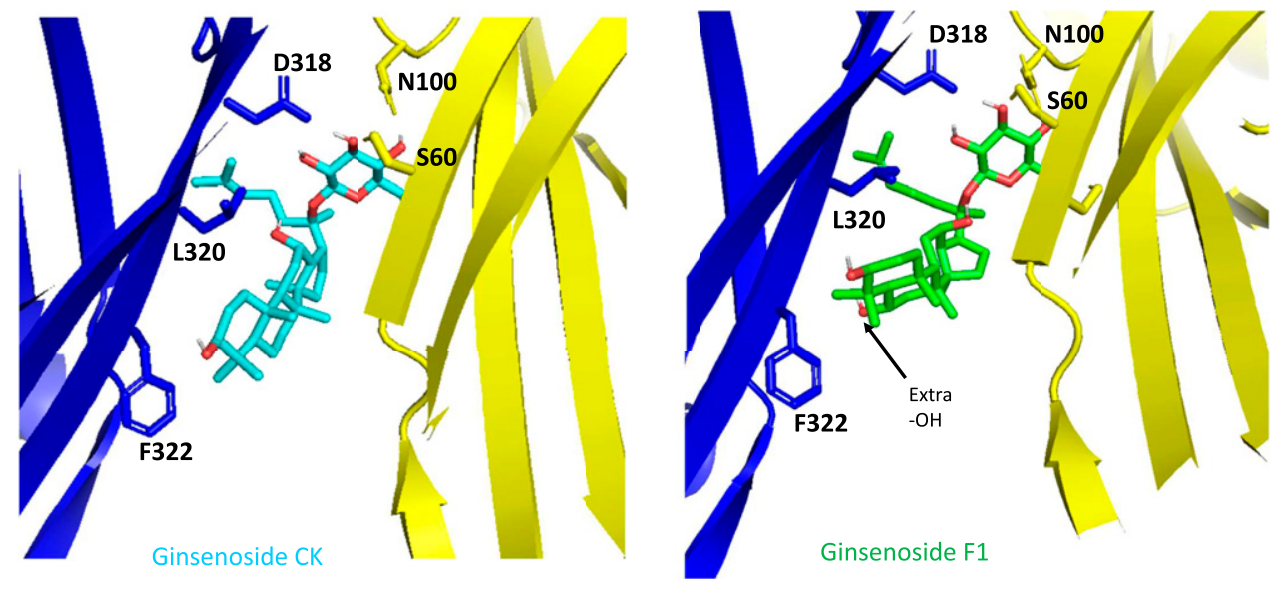

Fig. 4. Molecular docking of ginsenoside$\mathrm{CK}$ and ginsenoside-F1 to the central vestibule pocket of $\mathrm{hP} 2 \mathrm{X} 7$. GinsenosideCK (cyan) docked into the central vestibule in the ATP-bound homology model of human P2X7 and right, ginsenoside-F1 (green) docked into the same site. Side chains of amino acid residues of hP2X7 implicated in interactions are shown. 
A
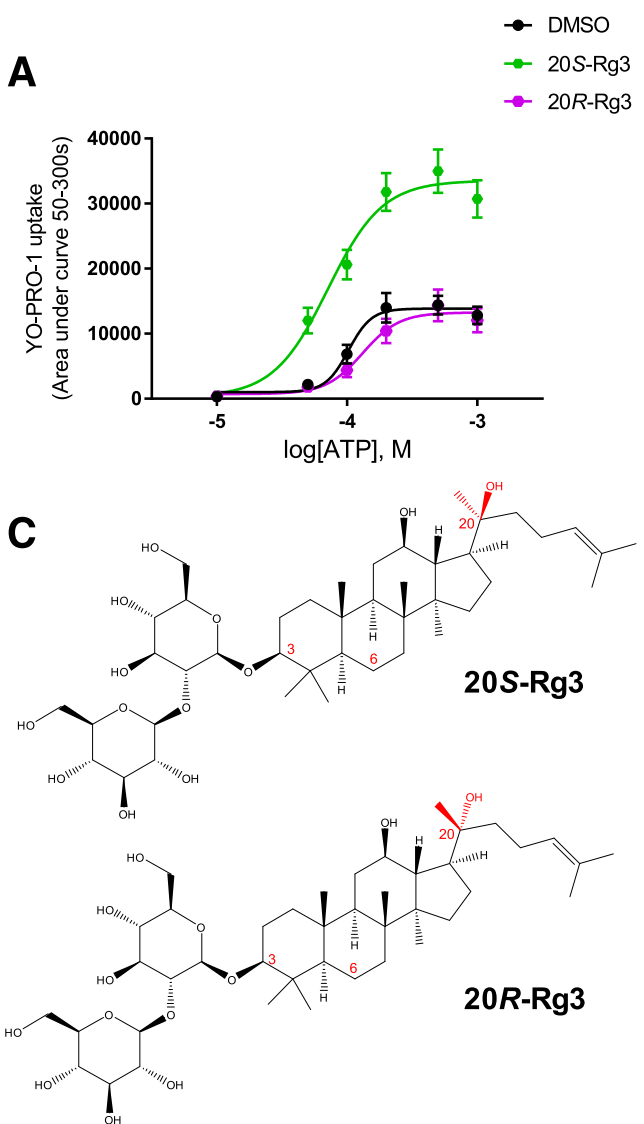

- 20S-Rg3

20R-Rg3
B

$\rightarrow$ DMSO
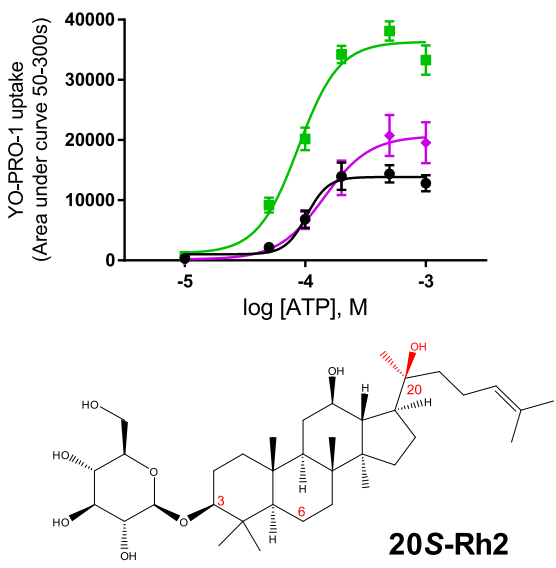

20S-Rh2

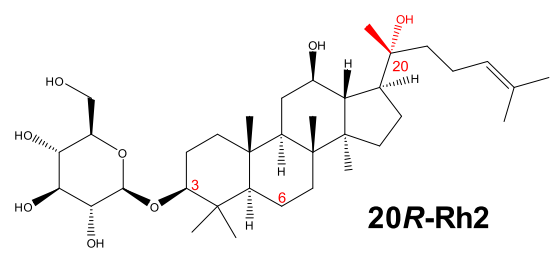

Fig. 5. Diastereoisomers of ginsenosides have different activity at hP2X7. (A) Doseresponse curves to ATP in the presence of vehicle (DMSO), 20(S)-ginsenosideRg3, or 20(R)-ginsenoside-Rg3 (10 $\mu \mathrm{M})$. (B) Dose-response curves to ATP in the presence of vehicle (DMSO), ginsenoside$20(S)$-Rh2, or ginsenoside-20(R)-Rh2 (10 $\mu \mathrm{M})$. Data are collated from three independent experiments and are means \pm S.D. The same DMSO data are shown in both plots. (C) Chemical structures of ginsenoside-Rg3 and ginsenoside-Rh2 are shown with the stereo-centers highlighted in red. abolished in cells pretreated with either AZ11645373 $(10 \mu \mathrm{M})$ or AZ10606120 (10 $\mu \mathrm{M})$, suggesting that the ginsenoside-CK potentiated response is solely mediated by P2X7 with no involvement of other purinergic receptors. Using the same protocol we investigated whether we could verify active and inactive glycosides as PAMs and confirmed that ginsenosideF2, ginsenoside-Rd, and ginsenoside-20(S)-Rg3 all potentiated the ATP-induced calcium response in THP-1 cells and that this potentiation was abolished by pretreatment with a P2X7-selective antagonist (Fig. 7C).

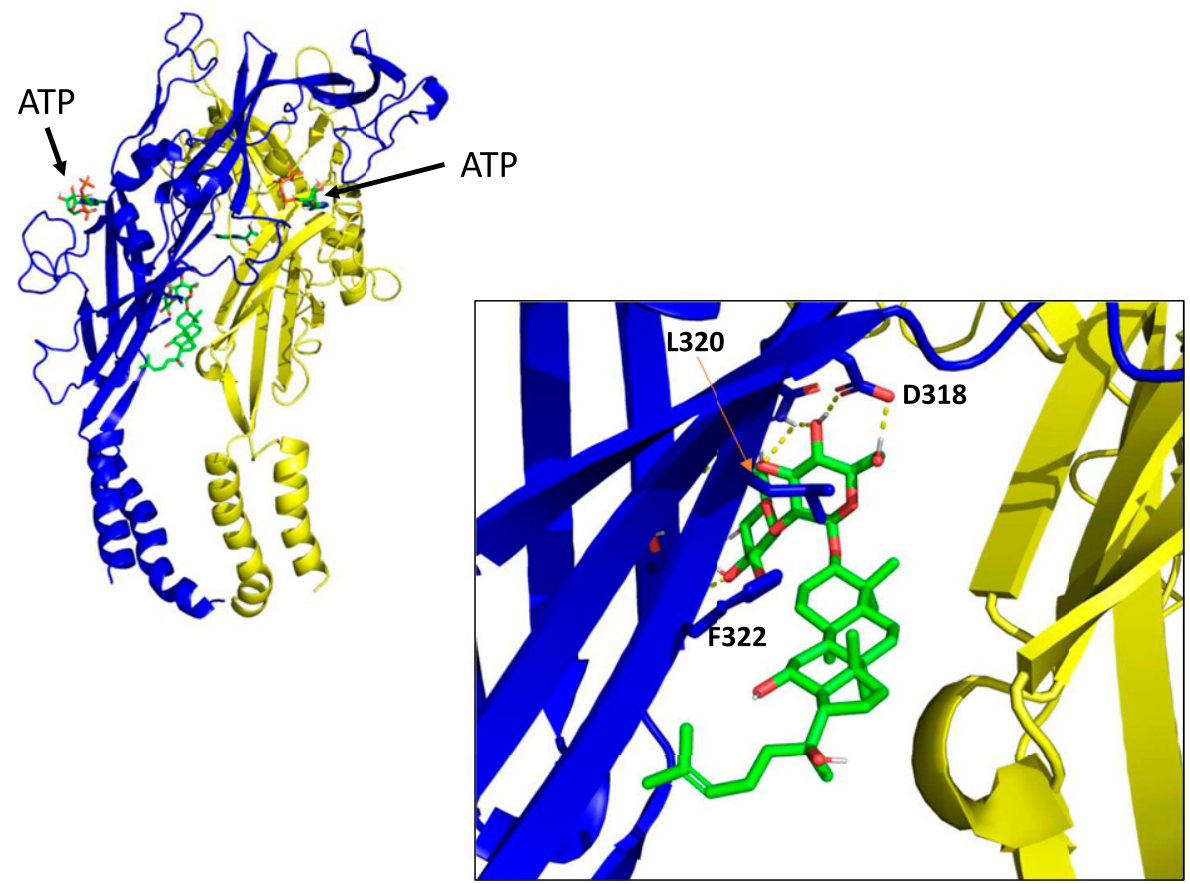

Fig. 6. Induced-fit docking of $20 \mathrm{~S}-\mathrm{Rg} 3$ at human P2X7 ginsenoside-20(S)-Rg3 (green) docked into the central vestibule site in a homology model of ATP-bound human P2X7 (open state). The predicted orientation of the stereocentre $\mathrm{C}-20$ is such that the $-\mathrm{OH}$ is pointing away from the hydrophobic face of the binding site, thus minimizing any repulsive interactions. Key side chains of residues D318, L320, and F322 are indicated. 
A

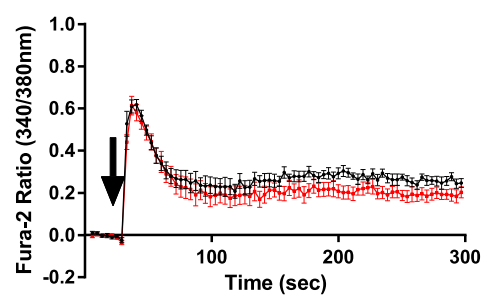

- ATP

$\rightarrow$ AZ116 ATP

B

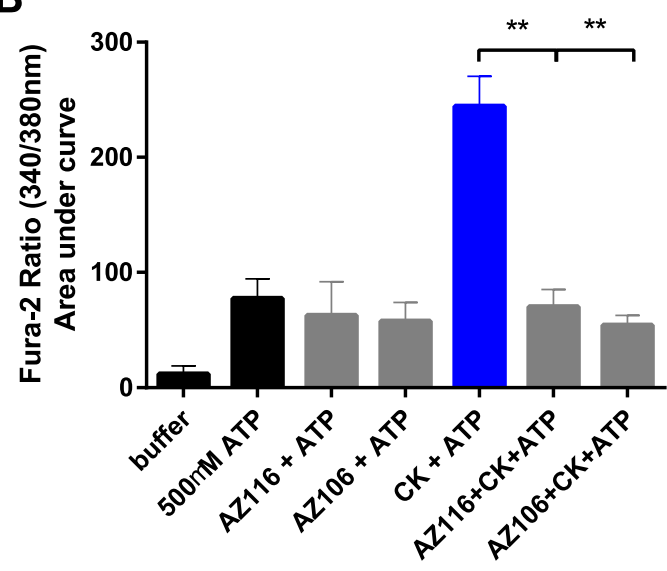

D

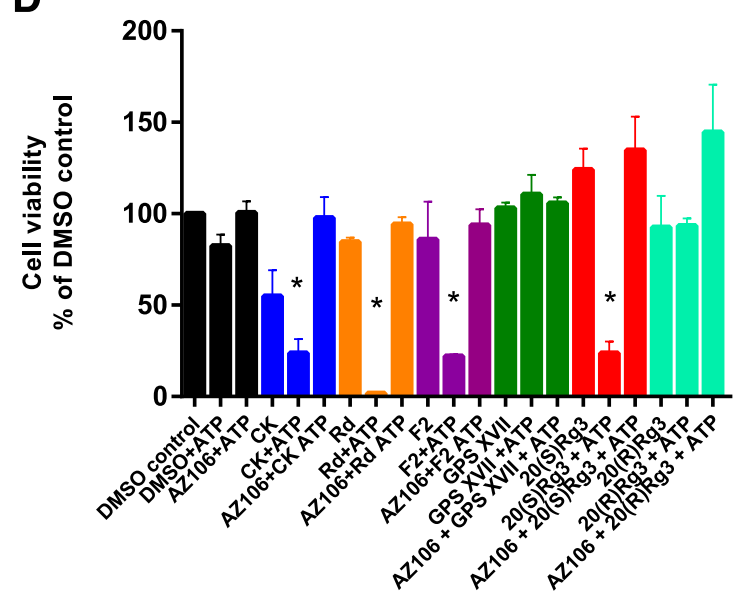

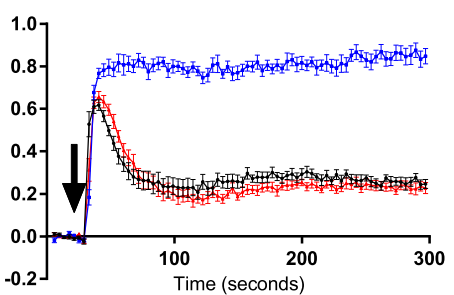

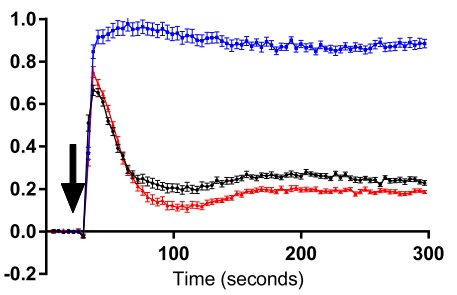

$\rightarrow$ ATP

$\rightarrow$ CK and ATP

- AZ116 ATP and CK
- ATP

- CK and ATP

- AZ106 CK and ATP

C

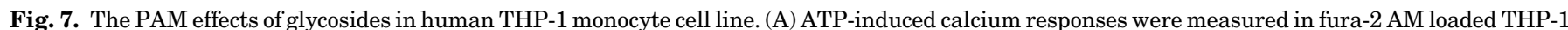

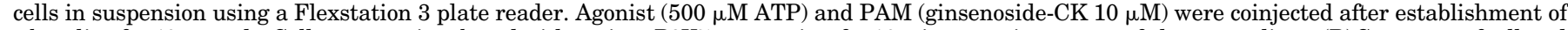

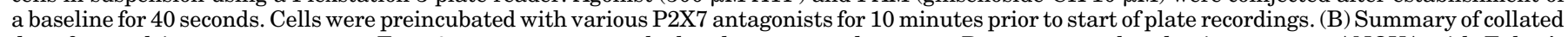

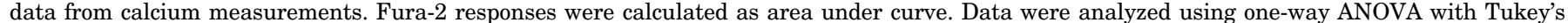

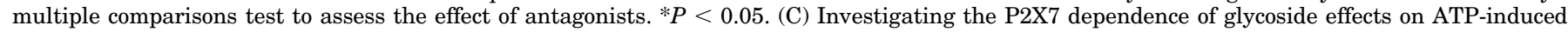

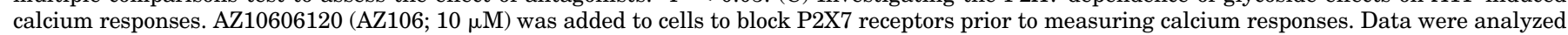

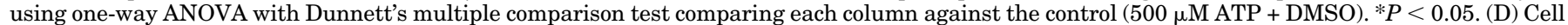

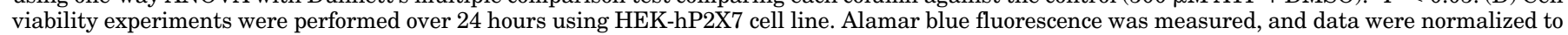

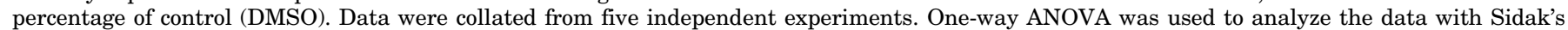

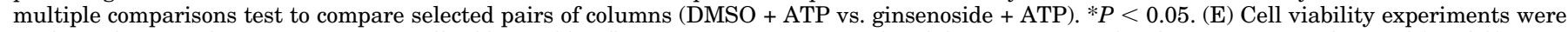

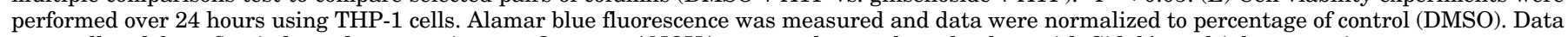

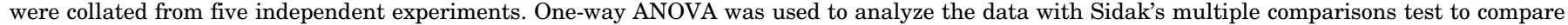
selected pairs of columns (DMSO + ATP vs. ginsenoside + ATP). ${ }^{*} P<0.05$.

We then determined if the identified active PAMs ginsenosideF2, 20(S)-Rg3, and gypenoside XVII could enhance P2X7dependent cell death in the highly expressing HEK-hP2X7 model using ginsenoside-CK as a positive control. Both ginsenoside-F2 and $20(S)$-Rg3 could reduce cell viability over 24 hours when applied in combination with ATP, and this was prevented by pretreatment with AZ10606120 (Fig. 7D). However, neither gypenoside XVII nor 20(R)-Rg3 in combination with ATP 
affected cell viability (Fig. 7D). In THP-1 cells, treatment with $500 \mu \mathrm{M}$ ATP significantly reduced cell viability to $73.3 \% \pm$ $14.4 \%$ of control (Fig. 7E), although this may not be P2X7dependent due to the lack of effect of AZ10606120. Only ginsenoside-CK could significantly enhance this ATP-induced cell death in THP-1 cells (Fig. 7E).

\section{Discussion}

In summary, we have demonstrated that the chemical requirements for positive allosteric modulators at P2X7 appear to be quite stringent. Dose-response experiments demonstrate that ginsenoside-CK and ginsenoside-Rd have two effects on ATP-induced responses at hP2X7 (Fig. 1B): an increase in the maximum response (a type I PAM effect) and a shift in the dose-response curve to the left (a type II PAM effect), thereby enhancing the maximum effect of the agonist and reducing the $\mathrm{EC}_{50}$ value. Our previous work has shown ginsenoside-CK, a triterpenoid glycoside with one glucose attachment, to have the best PAM effect at human P2X7. Here we show that ginsenoside-F2 and ginsenoside-20(S)-Rg3 have equivalent PAM activity at P2X7. We investigated a range of glycosides with different numbers of carbohydrate groups attached, and alternative sugar groups to glucose. Of these, gypenoside XVII showed the best PAM activity at P2X7. This glycoside is a dammarene glycoside found in Panax species, typically Panax notoginseng (Sakah et al., 2013). Gypenoside XVII has a single glucose attached at C-3 and two glucose groups attached at C-20 [a $\beta$-D-glucopyranosyl(1-6)- $\beta$-D-glucopyranoside]. It is very similar in structure to stevenleaf (also known as gypenoside IX; Table 1), but the sugar attachments on C-20 are different. Stevenleaf has a glucose-xylose disaccharide attached at C-20, whereas in gypenoside XVII, this is a glucose-glucose disaccharide. The reduced activity of stevenleaf (Supplemental Fig. 1) compared with gypenoside XVII suggests that there is a preferential requirement for glucose within the binding pocket on P2X7. Similarly, esculentoside A has a similar structure to ginsenoside-20(S)-Rg3 with a disaccharide on C-3, although this is composed of glucose-xylose, whereas Rg3 has a glucoseglucose disaccharide. Esculentoside A was inactive at P2X7 (Supplemental Fig. 1).

Most useful in terms of defining a structure-activity relationship for PAMs at P2X7 was the finding that modifications at C-6 were not tolerated. Ginsenoside-F1 showed no PAM activity at P2X7, and computational docking suggests that this additional hydroxyl on C-6 faces the hydrophobic side of the predicted binding pocket. This may be incompatible with binding to $\mathrm{P} 2 \mathrm{X} 7$ to produce effective potentiation of responses. We have not performed a binding assay to determine if this lack of activity equates to a lack of binding to P2X7 rather than equivalent binding and a lack of effect. Currently there are no suitably labeled ginsenosides available as probes, and this is something we shall explore in future studies. We also discovered that the stereochemistry of $20(S)$ versus $20(R)$ in ginsenoside-Rg3 and ginsenoside-Rh2 have a clear effect on PAM activity with only the $20(S)$ diastereomers retaining good PAM activity. Again, computational docking suggests this C-20 hydroxyl group to be close to the highly hydrophobic side of the predicted binding pocket, and we hypothesize that this is important in correct positioning leading to effective potentiation of ATP-dependent responses.
Regarding the differences in the terpenoid backbone, several of the compounds we selected for testing contained carbohydrate at either end of the molecule but did not contain the dammarene scaffold. For example, daucosterol is a monosaccharide (glucose) but contains a sitosterol-like scaffold. This includes a double bond that changes the shape of the scaffold and this has a detrimental effect on activity at P2X7. The key elements to the structure-activity relationship for glycosides at P2X7 are summarized in Fig. 8 showing two predicted binding modes for glycosides. Figure 8A shows the ginsenoside-CK binding mode with C-20 glucose inserted into the binding pocket. Figure $8 \mathrm{~B}$ shows the inverted mode for ginsenosides with C-3 glucose inserted into the binding pocket. Substitutions are tolerated on C-3 and C-20, but glucose is the preferred sugar attachment. Monosaccharides have higher activity than most disaccharides, and no substitution is tolerated at C-6 (Fig. 8A). In the inverted mode, the stereochemistry of the hydroxyl group on C-20 is critical for activity (Fig. 8B). In both cases the dammarene scaffold is favored over a sitosterol scaffold.

It is important to perform full dose-response analysis to determine those compounds that have PAM effects at P2X7. Saikosaponin A and solanine could increase the ATP-induced dye uptake in the screen on HEK-hP2X7 cells (Fig. 2A), but further investigation showed that these compounds had a toxic effect on the cells, inducing YO-PRO-1 dye uptake in the absence of ATP or the absence of the P2X7 receptor (Fig. 2). Saikosaponins have been linked to multiple biologic effects including induction of apoptosis ( $\mathrm{Li}$ et al., 2018).

Several groups have demonstrated endogenous steroidal compounds to have positive allosteric modulator activity at P2X receptors. Dehydroepiandrosterone and progesterone can potentiate rodent P2X2 receptors (De Roo et al., 2003, 2010), and $17 \beta$-ester derivatives of testosterone also potentiate rat P2X2 and P2X4 (Sivcev et al., 2019). None of these compounds had activity at P2X7 receptors. Lithocholic acid, a bile acid, has potentiating activity at P2X7 and P2X4 (rat) but inhibits rat P2X2 (Sivcev et al., 2020). This suggests there may exist endogenous regulators of P2X channel activity. It has been suggested that the bile acids may share a similar binding site to ivermectin, close to transmembrane domain 1 (Sivcev et al., 2020).

Positive allosteric modulation of P2X7 may be important in a number of contexts (Stokes et al., 2020). In this study we used the human monocytic cell line, THP-1, which has been used in other studies to investigate human $\mathrm{P} 2 \mathrm{X} 7$ responses (Stokes et al., 2006; Gadeock et al., 2010). Native P2X7 responses are small in this cell line, likely masked by the multitude of other purinergic ATP-responsive receptors present. P2X7-selective antagonists did not affect the ATPinduced calcium responses dramatically (Fig. 7A), yet we did see robust potentiation of the ATP-induced calcium response that was mediated by P2X7. The THP-1 cell line carries a lossof-function polymorphism in the C-terminus of P2X7, rs3751143, encoding the Glu496 > Ala amino acid substitution. Sequencing of exon 13 reveals heterozygosity for rs3751143 (Supplemental Fig. 2), confirming the work in Gadeock et al. (2012). Our data clearly show that P2X7 responses in THP-1 cells can be enhanced by ginsenoside$\mathrm{CK}$, and we can effectively rescue deficient P2X7 receptor responses in humans carrying this loss-of-function polymorphism. Ginsenoside-F2 and ginsenoside-Rd were also effective 


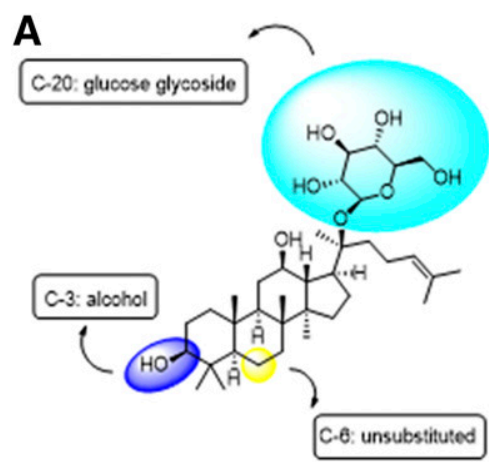

Ginsenoside CK

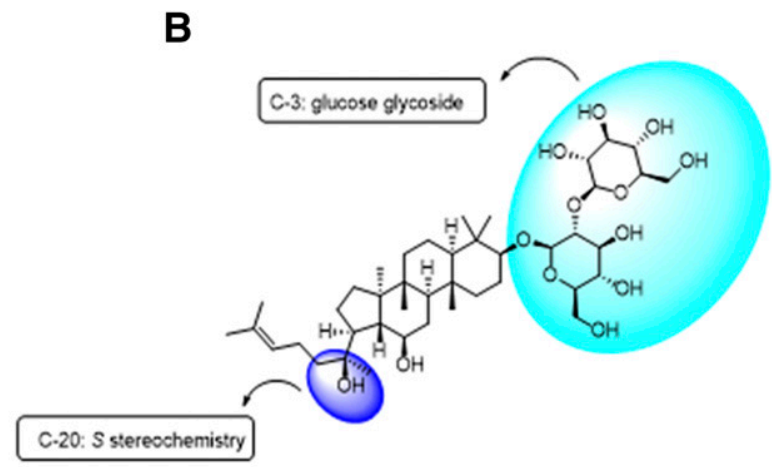

Ginsenoside (20S)-Rg3

Fig. 8. Proposed structure-activity relationship for glycosides acting as PAMs at P2X7. (A) The chemical structure of ginsenoside-CK is shown with important groups highlighted. Glucose attachment (cyan) is critical for activity at P2X7. C-6 substitutions are not tolerated (yellow). (B) The chemical structure of ginsenoside-20(S)-Rg3 is demonstrated with positioning relative to the P2X7 binding mode (inverted). The C-3 glucose attachments (cyan) face up into the binding pocket. The C-20 hydroxyl group shows that stereochemistry and positioning are critical for activity at P2X7.

at increasing P2X7 responses in THP-1 cells (Fig. 7), and ginsenoside-F1 was inactive. Extending this set of data that measures immediate P2X7 responses, we looked at P2X7mediated cell death first in HEK-hP2X7 cells using an Alamar blue cell viability assay. Ginsenoside-CK, ginsenoside-Rd, 20(S)-Rg3, and ginsenoside-F2 were very effective at enhancing cell death in combination with ATP; however, gypenoside XVII was unable to enhance P2X7-mediated cell death in this cell line (Fig. 7D). At this stage, it is unclear why this would be the case. Future investigations into the longevity of the potentiation may reveal that gypenoside XVII has a shorter duration of action than the ginsenosides, perhaps due to differential binding modes. Repeating this work in THP-1 cells showed that only ginsenoside-CK was effective at potentiation of cell death induced by ATP (Fig. 7E). This may be due to the lower level of expression of P2X7 in THP-1 cells, the expression of other purinergic receptors that may bind ginsenosides such as P2X4, or simply due to duration of action. It is also conceivable that monocytes may release factors such as glycosidase enzymes that can degrade ginsenosides.

Other compounds can act as positive allosteric modulators of P2X7 (Stokes et al., 2020), including clemastine (Nörenberg et al., 2011), tenidap (Sanz et al., 1998), ivermectin (Nörenberg et al., 2012), and polymyxin B (Ferrari et al., 2004). These bear no structural similarities to ginsenosides, although ivermectin is a glycoside, containing two oleandrose sugars. As yet nothing is known about the potential site of action of ivermectin on $\mathrm{P} 2 \mathrm{X} 7$, although on $\mathrm{P} 2 \mathrm{X} 4$ the binding site is proposed to be close to transmembrane domain 1 (Asatryan et al., 2010, Samways et al., 2012). In terms of therapeutic relevance of PAMs acting at P2X7, most exciting is the potential to enhance the microbicidal activity of immune cells. P2X7 has been implicated in regulation of pathogen killing, particularly those residing in intracellular locations such as mycobacteria and parasites (Di Virgilio et al., 2017, Savio et al., 2018). The mechanisms by which P2X7 contributes to reduction of pathogen burden are not yet well understood but could involve cytokine/mediator secretion, reactive oxygen species generation, or host cell apoptosis. We recently showed that potentiation of $\mathrm{P} 2 \mathrm{X} 7$ responses with ginsenoside-CK changed the type of cell death in the J774 macrophage cell line compared with high concentrations of ATP (Bidula et al., 2019a), and this could be relevant within an immune response. In models of infection, most of the work has been performed on P2X7-deficient mice to understand the role of this receptor within the host immune response. However, there has not been an in vivo study to investigate pharmacological targeting of P2X7 with a PAM. This idea has been tested in a zebrafish whole-animal study for Mycobacterium marinum infection using clemastine to potentiate $\mathrm{P} 2 \mathrm{X} 7$ (Matty et al., 2019), providing evidence that targeting P2X7 may be beneficial to control mycobacterial infections.

\section{Authorship Contributions}

Participated in research design: Ganesan, Bidula, Stokes.

Conducted experiments: Piyasirananda, Beekman, Stokes.

Performed data analysis: Piyasirananda, Beekman, Stokes.

Wrote, or contributed to writing of the manuscript: Piyasirananda, Beekman, Ganesan, Bidula, Stokes.

\section{References}

Areeshi MY, Mandal RK, Dar S, Wahid M, Khan ME, Panda AK, Jawed A, and Haque S (2015) P2X71513 A $>$ C polymorphism confers increased risk of extrapulmonary tuberculosis: a meta-analysis of case-control studies. Curr Genomics 17:450-458.

Asatryan L, Popova M, Perkins D, Trudell JR, Alkana RL, and Davies DL (2010) Ivermectin antagonizes ethanol inhibition in purinergic P2X4 receptors. $J$ Pharmacol Exp Ther 334:720-728.

Bidula S, Dhuna K, Helliwell R, and Stokes L (2019a) Positive allosteric modulation of P2X7 promotes apoptotic cell death over lytic cell death responses in macrophages. Cell Death Dis 10:882.

Bidula SM, Cromer BA, Walpole S, Angulo J, and Stokes L (2019b) Mapping a novel positive allosteric modulator binding site in the central vestibule region of human P2X7. Sci Rep 9:3231.

Chaves MM, Marques-da-Silva C, Monteiro AP, Canetti C, and Coutinho-Silva R (2014) Leukotriene B4 modulates P2X7 receptor-mediated Leishmania amazonensis elimination in murine macrophages. J Immunol 192:4765-4773.

Chaves MM, Sinflorio DA, Thorstenberg ML, Martins MDA, Moreira-Souza ACA Rangel TP, Silva CLM, Bellio M, Canetti C, and Coutinho-Silva R (2019) Noncanonical NLRP3 inflammasome activation and IL-1 $\beta$ signaling are necessary to L. amazonensis control mediated by P2X7 receptor and leukotriene B4. PLoS Pathog 15:e1007887.

De Roo M, Boué-Grabot E, and Schlichter R (2010) Selective potentiation of homomeric P2X2 ionotropic ATP receptors by a fast non-genomic action of progesterone. Neuropharmacology 58:569-577.

De Roo M, Rodeau JL, and Schlichter R (2003) Dehydroepiandrosterone potentiates native ionotropic ATP receptors containing the P2X2 subunit in rat sensory neurones. J Physiol 552:59-71.

Di Virgilio F, Dal Ben D, Sarti AC, Giuliani AL, and Falzoni S (2017) The P2X7 receptor in infection and inflammation. Immunity 47:15-31

Fairbairn IP, Stober CB, Kumararatne DS, and Lammas DA (2001) ATP-mediated killing of intracellular mycobacteria by macrophages is a P2X(7)-dependent process inducing bacterial death by phagosome-lysosome fusion. $J$ Immunol 167: 3300-3307.

Fernando SL, Saunders BM, Sluyter R, Skarratt KK, Goldberg H, Marks GB, Wiley JS, and Britton WJ (2007) A polymorphism in the P2X7 gene increases susceptibility to extrapulmonary tuberculosis. Am J Respir Crit Care Med 175:360-366. 
Ferrari D, Pizzirani C, Adinolfi E, Forchap S, Sitta B, Turchet L, Falzoni S, Minelli M, Baricordi R, and Di Virgilio F (2004) The antibiotic polymyxin B modulates $\mathrm{P} 2 \mathrm{X} 7$ receptor function. J Immunol 173:4652-4660.

Friesner RA, Banks JL, Murphy RB, Halgren TA, Klicic JJ, Mainz DT, Repasky MP, Knoll EH, Shelley M, Perry JK, et al. (2004) Glide: a new approach for rapid, accurate docking and scoring. 1. Method and assessment of docking accuracy. J Med Chem 47:1739-1749.

Gadeock S, Tran JNSN, Georgiou JG, Jalilian I, Taylor RM, Wiley JS, and Sluyter R (2010) TGF- $\beta 1$ prevents up-regulation of the P2X7 receptor by IFN- $\gamma$ and LPS in leukemic THP-1 monocytes. Biochim Biophys Acta 1798:2058-2066.

Gadeock S, Pupovac A, Sluyter V, Spildrejorde M, and Sluyter R (2012) P2X7 receptor activation mediates organic cation uptake into human myeloid leukaemic KG-1 cells. Purinergic Signal 8:669-676.

Giuliani AL, Sarti AC, Falzoni S, and Di Virgilio F (2017) The P2X7 receptor-interleukin-1 liaison. Front Pharmacol 8:123.

Hackos DH and Hanson JE (2017) Diverse modes of NMDA receptor positive allosteric modulation: mechanisms and consequences. Neuropharmacology 112:34-45.

Halgren T (2007) New method for fast and accurate binding-site identification and analysis. Chem Biol Drug Des 69:146-148.

Helliwell RM, ShioukHuey CO, Dhuna K, Molero JC, Ye JM, Xue CC, and Stokes L (2015) Selected ginsenosides of the protopanaxdiol series are novel positive allosteric modulators of P2X7 receptors. Br J Pharmacol 172:3326-3340.

Jacobson MP, Pincus DL, Rapp CS, Day TJ, Honig B, Shaw DE, and Friesner RA (2004) A hierarchical approach to all-atom protein loop prediction. Proteins $\mathbf{5 5}$ 351-367.

Li X, Li X, Huang N, Liu R, and Sun R (2018) A comprehensive review and perspectives on pharmacology and toxicology of saikosaponins. Phytomedicine $\mathbf{5 0}$ 73-87.

Matty MA, Knudsen DR, Walton EM, Beerman RW, Cronan MR, Pyle CJ, Hernandez RE, and Tobin DM (2019) Potentiation of P2RX7 as a host-directed strategy for control of mycobacterial infection. eLife 8:e39123.

Miller CM, Zakrzewski AM, Robinson DP, Fuller SJ, Walker RA, Ikin RJ, Bao SJ, Grigg ME, Wiley JS, and Smith NC (2015) Lack of a functioning P2X7 receptor leads to increased susceptibility to toxoplasmic ileitis. PLoS One 10:e0129048.

Moreira-Souza ACA, Almeida-da-Silva CLC, Rangel TP, Rocha GDC, Bellio M Zamboni DS, Vommaro RC, and Coutinho-Silva R (2017) The P2X7 receptor mediates Toxoplasma gondii control in macrophages through canonical NLRP3 inflammasome activation and reactive oxygen species production. Front Immunol 8:1257.

Nörenberg W, Hempel C, Urban N, Sobottka H, Illes P, and Schaefer M (2011) Clemastine potentiates the human P2X7 receptor by sensitizing it to lower ATP concentrations. J Biol Chem 286:11067-11081.
Nörenberg W, Sobottka H, Hempel C, Plötz T, Fischer W, Schmalzing G, and Schaefer M (2012) Positive allosteric modulation by ivermectin of human but not murine P2X7 receptors. Br J Pharmacol 167:48-66.

Placido R, Auricchio G, Falzoni S, Battistini L, Colizzi V, Brunetti E, Di Virgilio F, and Mancino G (2006) P2X(7) purinergic receptors and extracellular ATP mediate apoptosis of human monocytes/macrophages infected with Mycobacterium tuberculosis reducing the intracellular bacterial viability. Cell Immunol 244:10-18.

Qi LW, Wang CZ, and Yuan CS (2011) Ginsenosides from American ginseng: chemical and pharmacological diversity. Phytochemistry 72:689-699.

Sakah KJ, Wang T, Liu L, Chen Y, Han L, and Zhang Y (2013) Eight darmarane-type saponins isolated from the roots of Panax notoginseng. Acta Pharm Sin B 3 381-384.

Samways DS, Khakh BS, and Egan TM (2012) Allosteric modulation of Ca2+ flux in ligand-gated cation channel (P2X4) by actions on lateral portals. J Biol Chem 287: 7594-7602.

Sanz JM, Chiozzi P, and Di Virgilio F (1998) Tenidap enhances P2Z/P2X7 receptor signalling in macrophages. Eur J Pharmacol 355:235-244.

Savio LEB, de Andrade Mello P, da Silva CG, and Coutinho-Silva R (2018) The P2X7 receptor in inflammatory diseases: angel or demon? Front Pharmacol 9:52.

Sivcev S, Slavikova B, Ivetic M, Knezu M, Kudova E, and Zemkova H (2020) Lithocholic acid inhibits $\mathrm{P} 2 \mathrm{X} 2$ and potentiates $\mathrm{P} 2 \mathrm{X} 4$ receptor channel gating. J Steroid Biochem Mol Biol 202:105725.

Sivcev S, Slavikova B, Rupert M, Ivetic M, Nekardova M, Kudova E, and Zemkova H (2019) Synthetic testosterone derivatives modulate rat P2X2 and P2X4 receptor channel gating. $J$ Neurochem 150:28-43.

Stokes L, Bidula S, Bibič L, and Allum E (2020) To inhibit or enhance? Is there a benefit to positive allosteric modulation of P2X receptors? Front Pharmacol 11: 627.

Stokes L, Jiang LH, Alcaraz L, Bent J, Bowers K, Fagura M, Furber M, Mortimore M, Lawson M, Theaker J, et al. (2006) Characterization of a selective and potent antagonist of human P2X(7) receptors, AZ11645373. Br J Pharmacol 149:880-887.

Wei X, Chen J, Su F, Su X, Hu T, and Hu S (2012) Stereospecificity of ginsenoside Rg3 in promotion of the immune response to ovalbumin in mice. Int Immunol 24: 465-471.

Yang WZ, Hu Y, Wu WY, Ye M, and Guo DA (2014) Saponins in the genus Panax L. (Araliaceae): a systematic review of their chemical diversity. Phytochemistry 106: $7-24$.

Address correspondence to: Dr. Leanne Stokes, School of Pharmacy, University of East Anglia, Norwich Research Park, Norwich NR4 7TJ, UK. E-mail: l.stokes@uea.ac.uk 